EPJ Web of Conferences 92,02086 (2015)

DOI: $10.1051 /$ epjconf/ 20159202086

(C) Owned by the authors, published by EDP Sciences, 2015

\title{
Design and CFD Simulation of the Drift Eliminators in Comparison with PIV Results
}

\author{
Jiř́ Stodůlka ${ }^{1, a}$, Rut Vitkovičová ${ }^{1}$ \\ ${ }^{1}$ CTU in Prague, FME, Department of Fluid Dynamics and Thermodynamics, Prague, Czech Republic
}

\begin{abstract}
Drift eliminators are the essential part of all modern cooling towers preventing significant losses of liquid water escaping to the enviroment. These eliminators need to be effective in terms of water capture but on the other hand causing only minimal pressure loss as well. A new type of such eliminator was designed and numerically simulated using CFD tools. Results of the simulation are compared with PIV visulisation on the prototype model.
\end{abstract}

\section{Introduction}

This paper deals with the simulation of the flow in the optimized cooling tower drift eliminator in order to obtain it's functional parameters. The flow is simulated using CFD code ANSYS Fluent and to validate these results, the PIV visualization method is used together with experimental pressure loss evaluation on the prototype model to have a direct comparison between flow fields.

\section{Drift eliminators}

Beside the water distribution system and cooling panels are the eliminators another important functional part of the cooling tower [1], whose arrangement is schematically described in the figure 2.1.

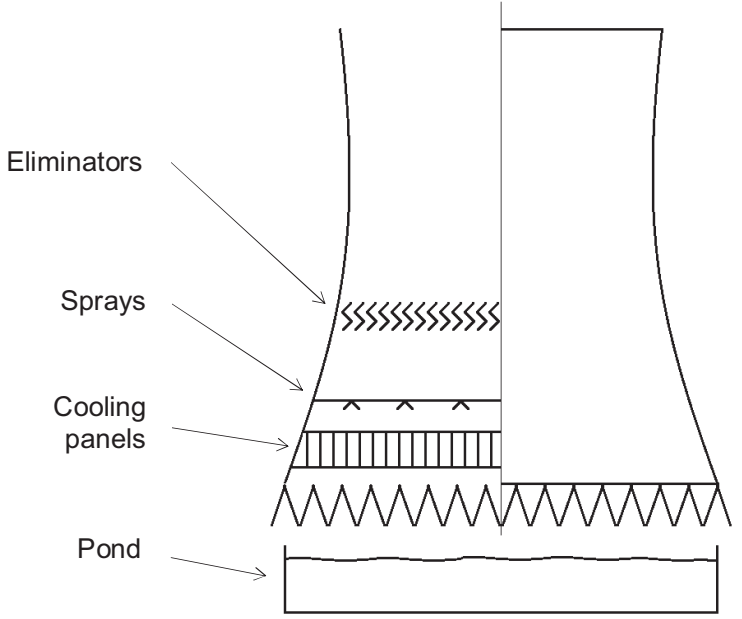

Figure 2.1. Natural draft cooling tower scheme.

\footnotetext{
${ }^{\mathrm{a}}$ Corresponding author: jiri.stodulka@fs.cvut.cz
}

The main purpose of these curved shape panels is to rapidly reduce the total mass flow carried away by air exiting the tower (drift) using their shape to change the flow direction causing enough inertia forces, so that the fluid droplets can't follow the air stream and hit the wall tickeling down as a film and drop back to the tower. The amount of water contained in both the form of vapor generated during the cooling process [2] and liquid droplets generated by condensation, but also very small diameter droplets drifted away from the spraying zone [3] escaping the tower, was estimated at approx. 0.001 $\mathrm{m}^{3} / \mathrm{hr} / \mathrm{MW}$ back in the year 2000 . This number may seem small, but taking into account high power outputs of modern energetic open recirculation systems, the total mass flow escaping is significant and that plays an important role in economical and also ecological function of the system. Local environment may suffer from high humidity values, constant fog clouds or hoarfrost. Operating water also contains treating chemicals such chlorine, bromine, the warm and moist air is very favourable condition for bacterial growth (Legionella pneumophila) and all these are then spread around the towers. To restrict the unpleasant side effects, the eliminator manufacturers have to guarantee nowadays to reduce the drift according to standards to maximum of $0.0005 \%$. The main escaping mass flow covers droplets in order of millimetres, which are heavy enough to be easily eliminated, but considering the smallest droplet diameters of around $1-10 \mu \mathrm{m}$, is this number still challenging.

The most commonly used types are the grid eliminators, made of thin profiled slats connected together. Example of such eliminator is shown in the figure 2.2. The material used for these eliminators is usually PVC, only for special systems with aggressive chemicals other materials like ceramics are used. 

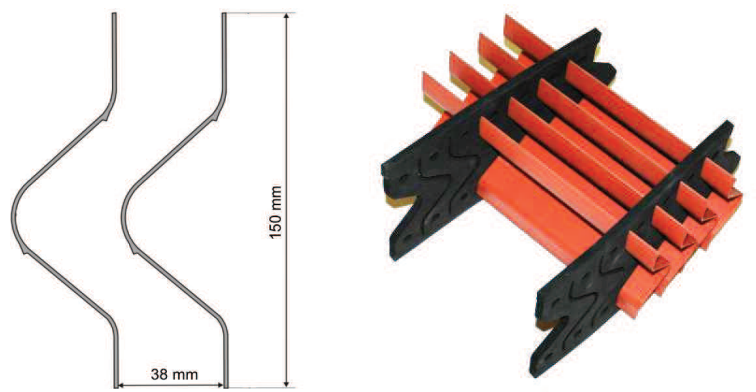

Figure 2.2. Grid type eliminator.

Another relatively new type is the eliminator with moisture recovery system. This type combines two principles; the first is the elimination of liquid phase similarly like the grid type eliminator and simultaneously a function of heat exchanger. These eliminators are bigger and hollow, to allow outer air with lower temperature pass through to cool down the escaping moist air ending in condensation of the moist air. A lot of attention is paid nowadays to improve the behaviour of these multifunctional eliminators in order to save more and more amount of water escaping from the evaporative cooling systems.

\section{Design}

Shape of every eliminator has to fulfil three main requirements. These are maximal efficiency in terms of caught water droplets, minimal pressure loss and minimal amount of used material. Unfortunately all three requirements are against each other, so it is necessary to find appropriate balance between them. In the first stage of design and consecutive optimization, it has been focused on the external flow. Internal channel just need to provide enough space for sufficient mass flow. Final shape of such eliminator row is in the figure 3.1.

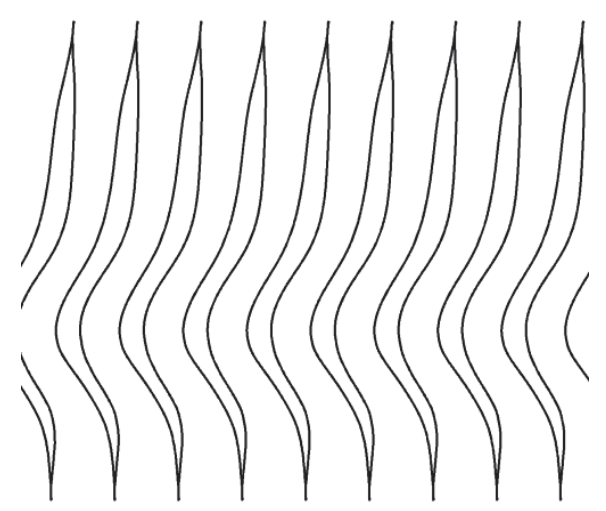

Figure 3.1. Eliminator shape.

The main ideas for eliminator design are to reach maximal velocities and maximal curvatures of the channel to achieve high inertia forces. On the other hand it is necessary to avoid noticeable flow separation to keep the pressure loss low. Big advantage of a hollow eliminator is the fact that thanks to space between the sides, shaping is much more unrestricted, unlike the grid type one, where any change one side immediately affects the flow on the other side.
In this case is the requirement for minimal used material neglected due to need for enough of condensation area. The overall height of the eliminator is $450 \mathrm{~mm}$, while distance between neighbouring ones is 60 $\mathrm{mm}$.

\section{Numerical simulation}

For the numerical simulations the commercial CFD code ANSYS Fluent was used. The character of the flow allows us to use only two dimensional model with periodic boundary condition for repeating eliminators. These facts significantly reduce the size of his case. Schematic view on the model and boundary conditions setup is in the figure 4.1. Velocity inlet values vary depending on concrete cooling tower conditions from 1 to $4 \mathrm{~m} / \mathrm{s}$ with the turbulent intensity of $2 \%$. Pressure at the outlet is set to be atmospheric.

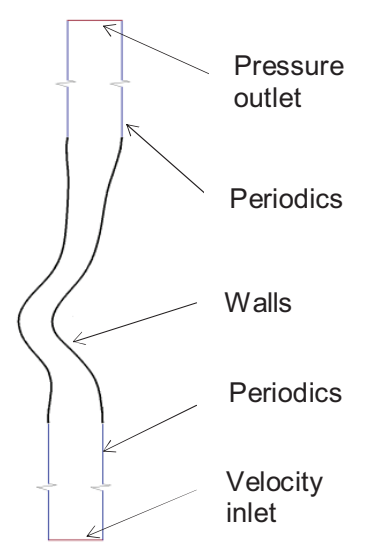

Figure 4.1. Computational model description.

Due to relatively simple geometry the mapped quad mesh was used for the whole domain with total count of approx. 100000 elements. Implemented sst k- $\omega$ turbulence model was used, what leads to demand for fine mesh close to the walls. The values of $\mathrm{Y}+$ oscillate around 0.5 for all velocity modes. The detail of the mesh is shown in figure 4.2 .

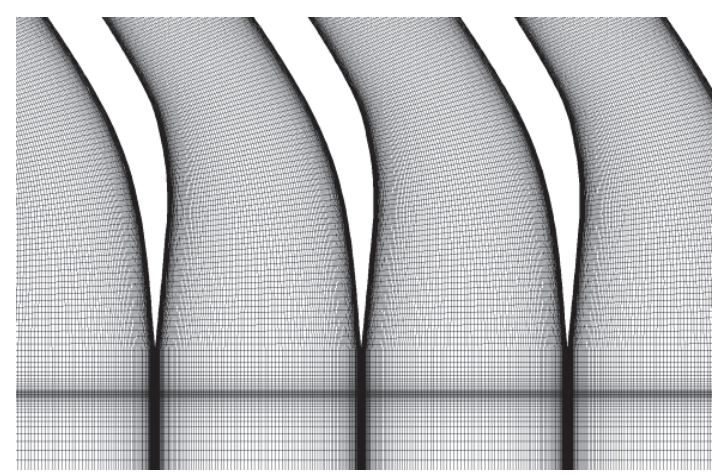

Figure 4.2. Quad computational mesh.

Results in form of velocity contours for inlet mode $2.6 \mathrm{~m} \mathrm{~s}^{-1}$ are shown in the figure 4.3. Except the trailing edge of the eliminator, no other separation occurs resulting in reasonable pressure loss. The flow is also significantly accelerated, so that droplets elimination can be expected in curved areas. 


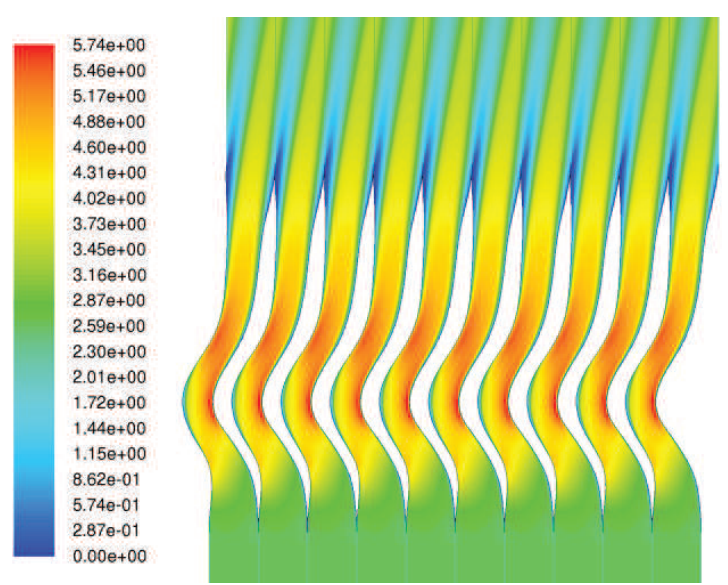

Figure 4.3. Contours of velocity magnitude $\left(\mathrm{m} \mathrm{s}^{-1}\right)$.

In terms of pressure loss, the maximal value for highest velocity mode $\left(4 \mathrm{~m} \mathrm{~s}^{-1}\right)$ is $5.9 \mathrm{~Pa}$, for the lowest mode $\left(1.5 \mathrm{~m} \mathrm{~s}^{-1}\right)$ is $1.2 \mathrm{~Pa}$.

\section{Experimental setup}

For experimental evaluation of the flow field the PIV method was used. Models of eliminator were placed in wind tunnel prepared for optical visualisation methods; see figure 5.1., with the laser sheet pointed into the mid section of the channel. To secure sufficient resolution of images even with this large scale model, the investigated area was subsequently traversed with the camera and the final image was composed from multiple local data sets. The inlet velocity was approximately $3.2 \mathrm{~m} \mathrm{~s}^{-1}$ and the CFD boundary condition for comparison simulation was adjusted according to that. Pressure loss was simply evaluated by pressure difference in front of and behind the eliminator in bigger classical open aerodynamic tunnel to minimize the affect of tunnel itself.

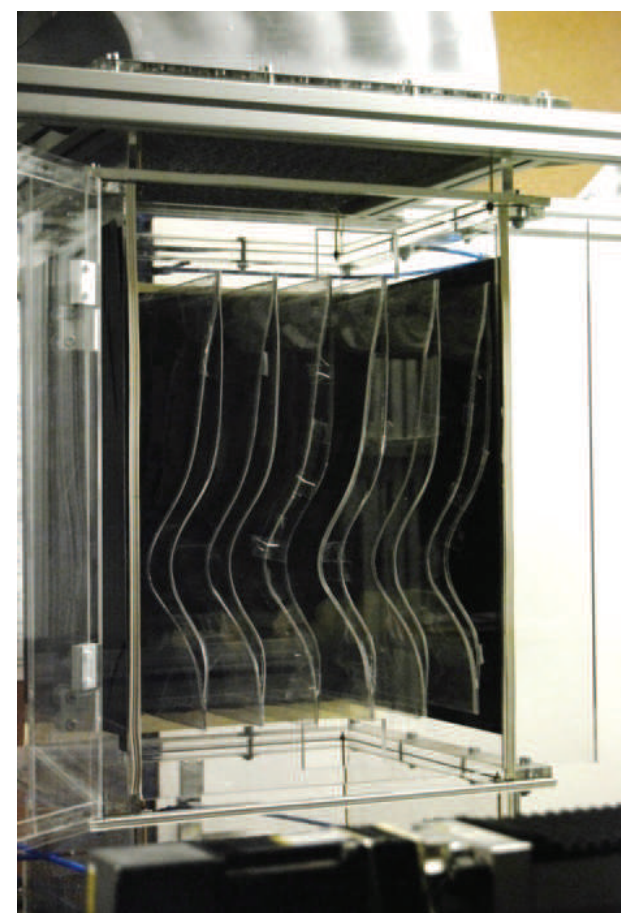

Figure 5.1. Experimental model.

\section{Comparison}

To obtain a direct comparison between experimental and numerical approach of the flow field evaluation, the data from both were analyzed using Matlab. Data in form of velocity component contours were plotted on the same grid and then subtracted from each other to see the differences. The evaluation of the x-velocity component is shown in the figure 6.1., in the upper left corner are the PIV results, in the lower left corner CFD and on the right side is the difference between them, CFD results subtracted from the PIV results.

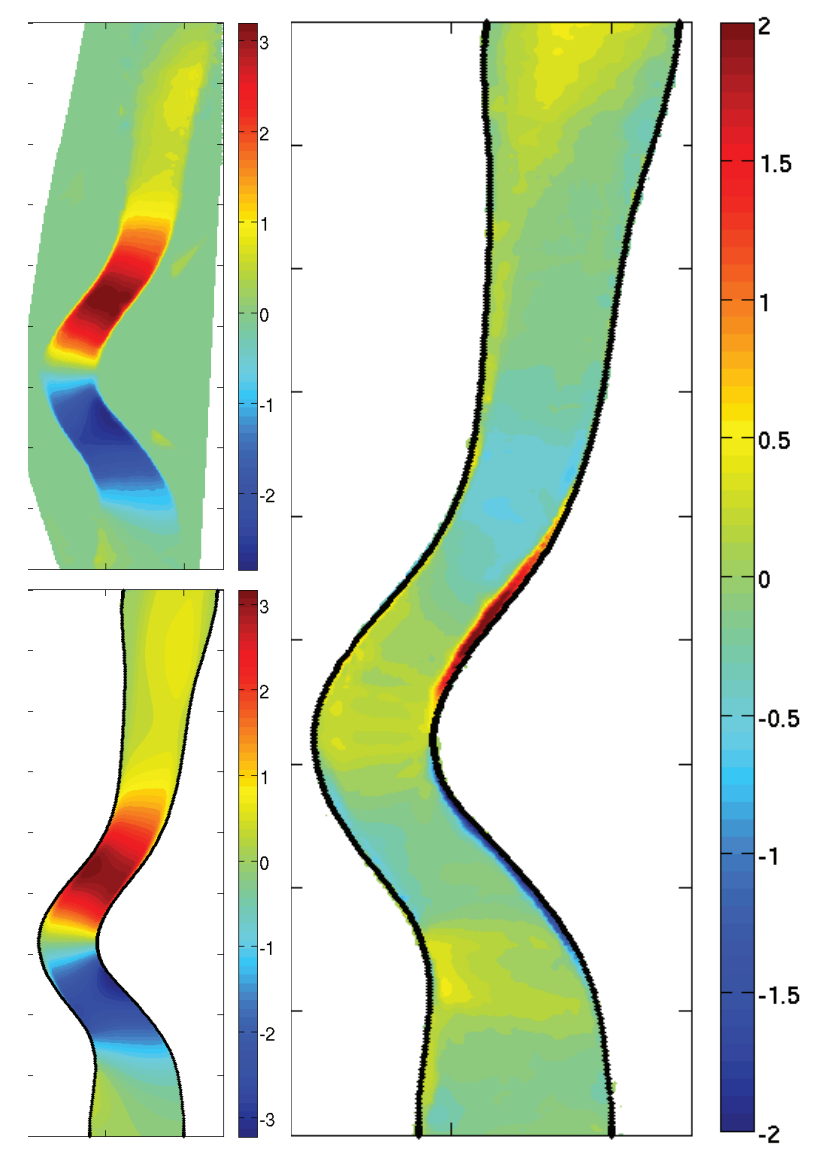

Figure 6.1. X-velocity comparison.

In the figure 6.2. are the same results for y-velocity component. From these figures is obvious a good accordance between these two approaches, at least in the main centre flow, where the difference ranges only in few tenths of $\mathrm{m} / \mathrm{s}$, what is also confirmed lower from velocity profiles. Near walls is the difference more dramatic, but that can be due to limits of the PIV near strongly reflective boundary and direct real and model geometry comparison. Major inconsistency appears only at the upper part of the eliminator, where mainly y-components exhibit significant disparity. That was probably caused by inconsistence between numerical and physical eliminator model and the finite dimension of the wind tunnel can play its role as well. However, this area is no more important in terms of the main function of the eliminator, while all possible droplet separation happens in the curved section. 


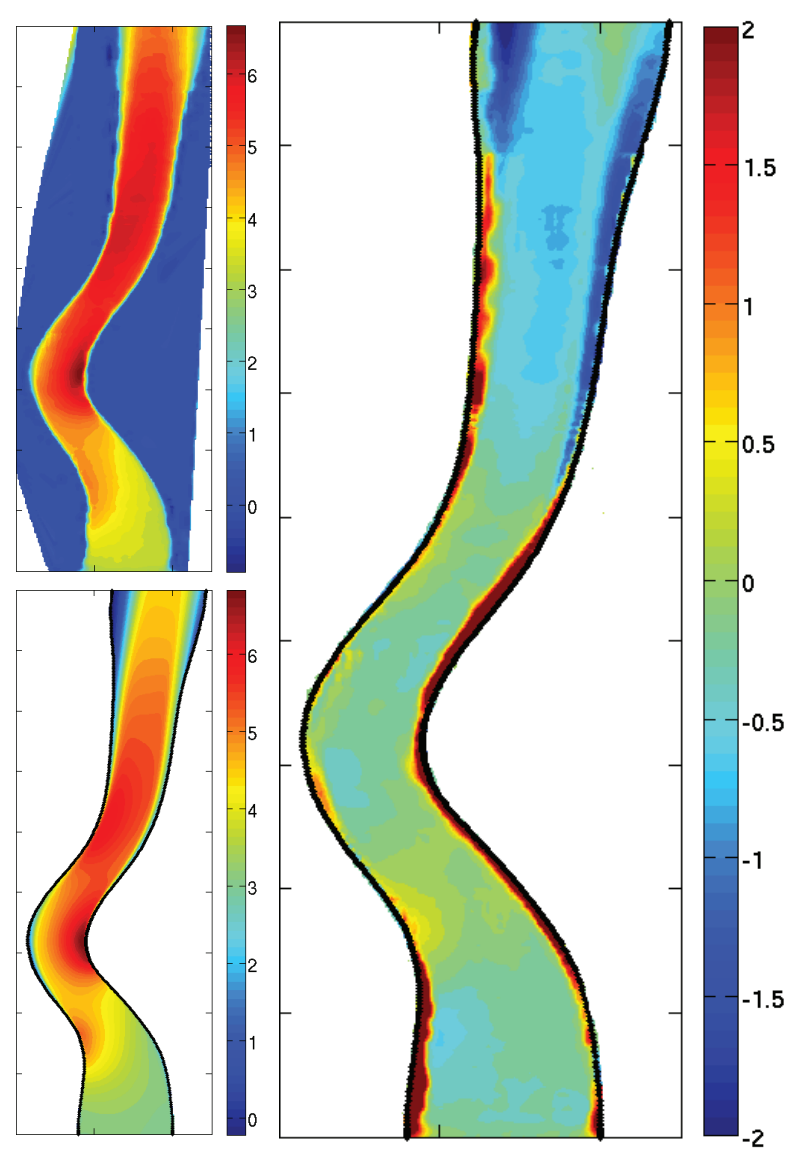

Figure 6.2. Y-velocity comparison.

In the figure 6.3. are the velocity profiles in three cross section areas, blue solid line represents numerical data, dashed the experimental. Except near wall values and minor oscillations of experimental data a good correspondence is obvious here.
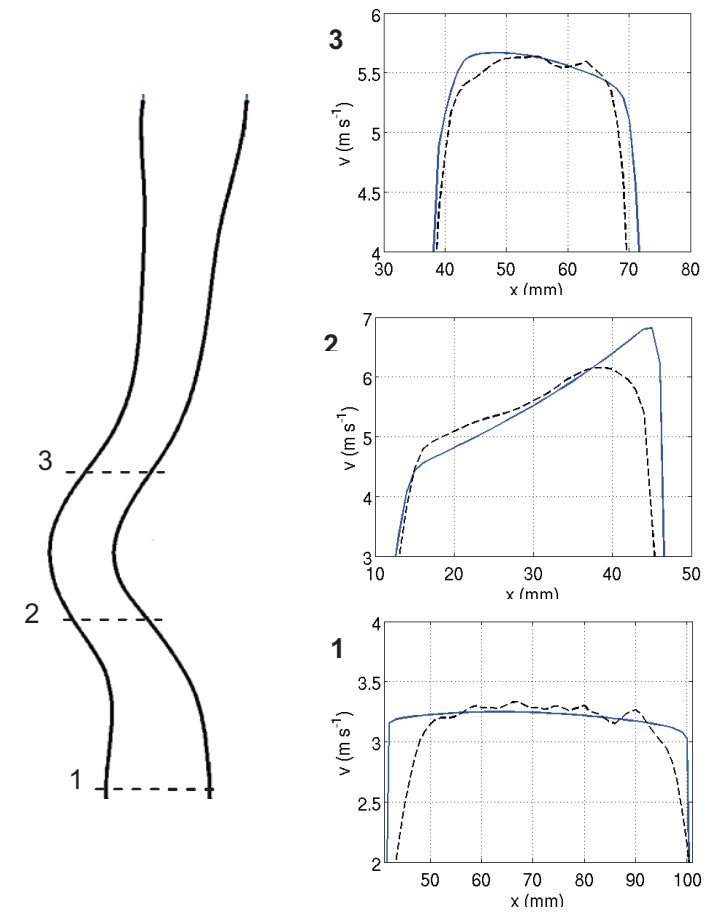

Figure 6.3. Velocity profiles.
Comparison of the pressure loss between both approaches is in the figure 6.4. Here it is necessary to take into account not just geometry differences, but also the error of measured quantities, especially for such low velocities and pressure values. Black points with error bars represent the experimantal evaluation and blue crosses the numerics.

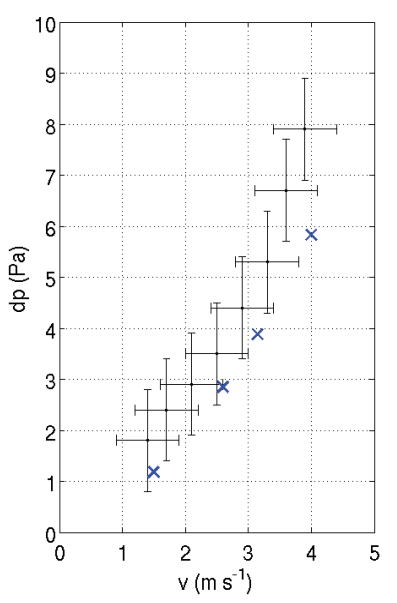

Figure 6.4. Pressure loss comparison.

Considering the geometry differeces, the CFD is slightly underpredicting in direct comparison, but the numerical results are still within the errorbars.

\section{Conclusions}

A direct comparison between numerical and experimental results of the flow through the cooling tower drift eliminator has been done in order to validate the CFD methods for this application. Drift eliminators are important part of the cooling system and severe requirements on them are expected in not too distant future. Modern CFD is a very fast and effective tool that can significantly help with design or optimization in this field and the reliability of this approach has been proved both in terms of flow field and pressure loss. Considering problematic exact geometry capture and setup, the results of this comparison are more than acceptable.

\section{References}

1. J. Čížek, L. Nováková, J. Nožička, Reducing Liquid Phase Drift from Cooling Towers, (Gradient, Prague 2009)

2. T. Hyhlík, EPJ Web of Conferences, 67, (2014), 02044

3. P. Vitkovič, V. Syrovátka, Journal of Flow Visualization and Image Processing, 16(4), (2009), pp. $367-375$

\section{Acknowledgement}

This work has been supported by Technology Agency of the Czech Republic under the project Advanced Technologies for Heat and Electricity Production TE01020036 